\title{
Systemic Treatment for Adults with Synovial Sarcoma
}

Ingrid M. E. Desar, MD, PhD ${ }^{1}$

Emmy D. G. Fleuren, $P h D^{2}$

Winette T. A. van der Graaf, MD, PhD ${ }^{1,3, *}$

\author{
Address \\ ${ }^{1}$ Department of Medical Oncology, Radboud University Medical Centre Nijmegen, \\ Nijmegen, The Netherlands \\ ${ }^{2}$ The Institute of Cancer Research, Sutton, UK \\ ${ }^{*}, 3$ The Institute of Cancer Research and the Royal Marsden NHS Foundation Trust, \\ London, UK \\ Email: winette.vandergraaf@icr.ac.uk
}

Published online: 7 March 2018

(C) The Author(s) 2018. This article is an open access publication

This article is part of the Topical Collection on Sarcoma

Keywords Synovial sarcoma • Chemotherapy · Targeted therapy · Immunotherapy

\section{Opinion Statement}

Synovial sarcoma (SS) is a rare, yet highly malignant, type of soft tissue sarcoma (STS), for which survival has not improved significantly during the past years. In this review, we focus on systemic treatment in adults. Compared to other STS, SS are relatively chemosensitive. Ifosfamide and ifosfamide combinations are active in different lines of treatment. In high-risk extremity and chest wall STS, neoadjuvant doxorubicin and ifosfamide has shown as much activity as high-dose ifosfamide. There are indications that combination chemotherapy with doxorubicin and ifosfamide in this setting improves outcome. In the first-line metastatic setting, combination treatment with doxorubicin and ifosfamide is a preferred option in fit patients, while in other patients, sequential doxorubicin and ifosfamide can be considered. In second and later lines, pazopanib and trabectedin have shown activity. Many new approaches to treat metastatic SS are currently under investigation, both preclinical as well as clinical, including other receptor tyrosine kinase inhibitors, epigenetic modulators, compounds interfering with DNA damage response (DDR), and immunotherapy.

\section{Introduction}

Soft tissue sarcomas (STS) are rare, malignant mesenchymal tumors that include over 70 different and highly diverse histological subtypes. Synovial sarcoma (SS) accounts for $5-10 \%$ of all STS $[1,2]$. SS is an intriguing disease as, unlike the majority of STS, it can occur at any age and everywhere in the body. The peak incidence is in the $30 \mathrm{~s}$ and it often presents in the extremities [3•]. The incidence of SS continued to increase from 0.906 to 
1.548 per 1,000,000 between 1983 and 2012 [4]. Despite its name, SS does not arise from the synovium, neither expresses synovial markers [5]. SS was initially described as a biphasic neoplasm comprising of both epithelial and uniform spindle cell components. SS is characterized by the presence of the pathognomonic $t(X ; 18)(\mathrm{p} 11.2 ; \mathrm{q} 11.2)$ translocation, involving a fusion of the SS18 (formerly SYT) gene on chromosome 18 to one of the synovial sarcoma $X$ (SSX) genes on chromosome X (usually SSX1 or SSX2), which is seen in more than $90 \%$ of SS and results in the formation of SS18-SSX fusion oncogenes [6].

For localized non high-risk disease, treatment consists of surgery, on indication combined with (neo)adjuvant radiotherapy. In about $50 \%$ of cases, metastases occur [7]. Interestingly, the prognosis of primary non metastasized SS is related to the age of the patient, with a much better relative survival in children compared to older patients, and more genomic instability with increasing age $[3 \bullet, 8]$. The 5 year overall survival (OS) for all SS is $60.5 \%$, but is largely age-dependent [4].

In most cases, metastases are localized in the lung $(80 \%)$, although metastases can arise in lymph nodes (up to $20 \%$ ), bone $(9.9 \%)$, and liver $(4.5 \%)$ as well [9•, 10]. Once metastasized, curative treatment is hardly achievable, with the exception of late and resectable oligometastatic disease and patients are treated with chemotherapy with a palliative intent. Compared to STS as a group, SS is more sensitive to certain chemotherapeutic agents $[9 \bullet, 11]$.

For long, STS have been clinically treated as one type of disease, and most chemotherapy trials included the majority of STS subtypes. The first attempt to address the differences in tumor behavior led to stratification for leiomyosarcomas, liposarcomas, SS, and the so-called other group and made use of the 3 - and 6-month progression-free rate (PFR) in second- and higher-line studies [12].

It is only in recent years that more sarcoma subtypespecific trials are undertaken, recognizing the large diversity in clinical behavior, biology, and genetic makeup of the different STS and appreciating the recent insights in more tumor-specific therapy. We here review the current standard of care for treatment of advanced and metastatic SS in adults and provide insights in the developments within the fields of targeted therapy and immunotherapy.

\section{Current pharmacological treatment options}

\section{Chemotherapy}

\section{(Neo)adjuvant chemotherapy}

The insights on (neo)adjuvant chemotherapy in STS have excellently been reviewed very recently $[13,14]$ and the most important trials are summarized in Table 1. In summary, in adults with localized STS of all localizations, chemotherapy in an adjuvant setting is not the standard of care, since many adjuvant STS trials, including SS, ultimately failed to prove overall survival benefit [20]. Neoadjuvant chemotherapy might be considered in specific situations, for example as induction therapy to enhance outcome of surgery in high-risk sarcoma of extremity and chest wall.

Recent data suggest that also DFS may benefit from this approach. In this respect, two studies in SS are worth mentioning.

A phase II trial exploring neoadjuvant treatment with doxorubicin $60 \mathrm{mg} /$ $\mathrm{m}^{2}$ and ifosfamide $10 \mathrm{~g} / \mathrm{m}^{2}$ for three neoadjuvant and two adjuvant courses in STS of the extremities, included 20 SS patients out of a total of 70 patients, and reported 2- and 5-year progression-free survival (PFS) rates of 75.7\% (95\% CI, $63.9-84.1 \%)$ and $63.8 \%(95 \% \mathrm{CI}, 51.3-73.9 \%)$, respectively. The 5-year OS was $82.6 \%$ (95\% CI, 71.3-89.7\%). Protocol treatments were completed in 74\% of the cases and toxicity was significant [17]. 
Table 1. (Neo)adjuvant chemotherapy

\begin{tabular}{|c|c|c|c|c|c|}
\hline (Neo)adjuvant treatment & Population & Phase & Total $n$ (SS) & Outcome & $\begin{array}{l}\text { Year of } \\
\text { publication }\end{array}$ \\
\hline $\begin{array}{l}\text { Histology-tailored } \\
\text { neoadjuvant } \\
\text { chemotherapy in } 5 \text { types } \\
\text { of STS. For SS: high-dose } \\
\text { ifosfamide }\left(14 \mathrm{~g} / \mathrm{m}^{2}\right. \\
\text { in } 14 \text { days) every } 28 \text { days } \\
\text { vs. epirubicine } \\
+ \text { ifosfamide } 9 \mathrm{~g} / \mathrm{m}^{2} \\
\text { in } 3 \text { days every } 21 \text { days } \\
{[15 \bullet \bullet]}\end{array}$ & $\begin{array}{l}\text { SS } \\
\text { LMS } \\
\text { mLPS } \\
\text { MPNST } \\
\text { UPS }\end{array}$ & III & $287(70)$ & $\begin{array}{l}\text { DFS } \\
\text { after } 46 \text { months } \\
\text { histology-tailored } \\
\text { chemotherapy } \\
38 \% \\
\text { vs. standard group } \\
46 \%, 0 \text { S } \\
89 \% \text { vs. } 64 \% \\
\text { (both significant, } \\
\text { median follow-up } \\
12.3 \text { months, } \\
70 \text { events) } \\
\text { Subgroup analysis: } \\
\text { standard } \\
\text { chemotherapy } \\
\text { favorable for SS } \\
\text { (HR 1.85, } 95 \% \text { CI } \\
\text { 0.65-5.22, } 17 / 70 \\
\text { events) }\end{array}$ & 2017 \\
\hline $\begin{array}{l}\text { Doxorubicin } 75 \mathrm{mg} / \mathrm{m}^{2} \text {, } \\
\text { ifosfamide } 5 \mathrm{~g} / \mathrm{m}^{2} \text {, } \\
\text { and lenograstim q } 3 \text { weeks for } \\
5 \text { cycles adjuvant } \\
\text { vs. active controle. } 73 \% \\
\text { also had radiotherapy [16] }\end{array}$ & STS & III & $351(40)$ & $\begin{array}{l}\text { Median OS } 12.4 \\
\text { vs. } 11.2 \text { years } \\
\quad \text { (not significant) } \\
5 \text { years EFS: } 52.9 \\
\text { vs. } 54.9 \% \\
5 \text { years } 0 \text { S } 67.8 \\
\text { vs. } 66.5 \%\end{array}$ & 2012 \\
\hline $\begin{array}{l}\text { Doxorubicin } 60 \mathrm{mg} / \mathrm{m}^{2} \text { and } \\
\text { ifosfamide } 10 \mathrm{~g} / \mathrm{m}^{2} \text { for } 3 \\
\text { neoadjuvant and } 2 \text { adjuvant } \\
\text { courses [17] }\end{array}$ & STS & II & $70(20)$ & $\begin{array}{l}2 \text { years PFS } 75.7 \% \\
5 \text { years PFS } 63.8 \% \\
5 \text { years } 0 S 82.6 \%\end{array}$ & 2015 \\
\hline $\begin{array}{l}\text { Doxorubicin } 37.5 \mathrm{mg} / \mathrm{m}^{2} \text { for } \\
2 \text { days and ifosfamide } 3 \mathrm{~g} / \mathrm{m}^{2} \\
\text { for } 3 \text { days followed by } 2 \text { cycles } \\
\text { of ifosfamide } 3 \mathrm{~g} / \mathrm{m}^{2} \text { for } \\
2 \text { days [18] }\end{array}$ & SS & II & 138 & $\begin{array}{l}3 \text { years EFS } 81.9 \% \\
5 \text { years EFS } 80.7 \% \\
3 \text { years OS } 97.2 \% \\
5 \text { years OS } 90.7 \%\end{array}$ & 2015 \\
\hline Not specified [19] & $\begin{array}{l}\text { SS stage } \\
\text { I-III }\end{array}$ & IV & $\begin{array}{l}544 \text { pts. } \\
\text { of whom } 131 \\
\text { received } \\
\text { chemotherapy }\end{array}$ & $\begin{array}{l}\text { Hazard ratio } \\
\text { chemotherapy for } \\
\text { OS: } \\
\text { - overall cohort: } \\
0.95 \\
(0.63-1.44) \\
\text { - stage I } 4.80 \\
(0.91-25.3) \\
\text { - stage IIA } 1.32 \\
(0.54-3.20) \\
\text { - stage IIB } 0.36 \\
(0.07-1.76)\end{array}$ & 2017 \\
\hline
\end{tabular}


Table 1. (Continued)

\begin{tabular}{|c|c|c|c|c|c|}
\hline \multirow[t]{2}{*}{ (Neo)adjuvant treatment } & Population & Phase & Total $n$ (SS) & Outcome & $\begin{array}{l}\text { Year of } \\
\text { publication }\end{array}$ \\
\hline & & & & $\begin{array}{l}\text { - stage III } 0.56 \\
(0.33-0.93), p= \\
0.028\end{array}$ & \\
\hline
\end{tabular}

SS synovial sarcoma, STS soft tissue sarcoma, MLPS myxoid liposarcoma, MPNST malignant peripheral nerve sheath tumor, UPS undifferentiated pleiomorphic sarcoma, DFS disease free survival, OS overall survival, PFS progression free survival, EFS event free survival

Results of a recent study in high-risk STS of extremity and chest wall, support the role of neoadjuvant combination chemotherapy, because of a gain in

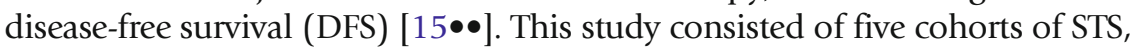
with an SS cohort including 70 patients. Patients were randomized 1:1 to three cycles of standard treatment consisting of ifosfamide $3 \mathrm{~g} / \mathrm{m}^{2}$ on days $1-3$ and epirubicin $60 \mathrm{mg} / \mathrm{m}^{2}$ on days 1-2 of every 21 days vs. histology-tailored chemotherapy, which was in SS high-dose ifosfamide $1 \mathrm{~g} / \mathrm{m}^{2}$ on days 1-14 of every 28 days [ $15 \bullet \bullet$. After a median follow-up of 12.3 months for the total study population $(n=287)$, the projected DFS at 46 months was $62 \%(95 \% \mathrm{CI}$ $48-77)$ in the standard chemotherapy group and $38 \%[21 \bullet \bullet, 22,23-26,27 \bullet$, $28 \bullet, 29,30-37,38 \bullet, 39,40-44,45 \bullet \bullet, 46,47,48,49 \bullet, 50,51,52 \bullet, 53,54]$ in the histotype-tailored chemotherapy group (stratified log-rank $p=0.004$; hazard ratio $2.00,95 \%$ CI $1.22-3.26 ; p=0.006$ ). Subgroup analysis indicated no preference for histology-tailored chemotherapy in the SS cohort (HR 1.85, 95\% CI 0.69-5.22). Final OS data are awaited.

Another risk-adapted approach had been included in a study of the European Pediatric Soft tissue sarcoma Study Group (EpSSG). SS patients < age of 21 years were treated in the EpSSG NRSTS 2005 trial, which included 138 children and adolescents with SS [18]. Treatment was tailored according to risk group. Low-risk patients ( $\mathrm{R} 0$ resection, tumor $\leq 5 \mathrm{~cm}$ in size) were treated with surgery alone, intermediate-risk patients ( $\mathrm{R} 0$ resection and tumor $>5 \mathrm{~cm}$ in size, or R1 resection) with three to six courses of chemotherapy \pm radiotherapy, and high-risk patients ( $\mathrm{R} 2$ resection, no resection at all, or N1) were treated with six cycles of chemotherapy, surgery, and radiotherapy. Chemotherapy consisted of a maximum of four cycles of doxorubicin $37.5 \mathrm{mg} / \mathrm{m}^{2}$ for 2 days and ifosfamide $3 \mathrm{~g} / \mathrm{m}^{2}$ for 3 days followed by two cycles of ifosfamide $3 \mathrm{~g} / \mathrm{m}^{2}$ for 2 days. After a median follow-up of 52 months, the 3 - and 5-year event-free survival (EFS) for all risk groups was 81.9 and $80.7 \%$, and the 3- and 5-years OS was 97.2 and $90.7 \%$, respectively. The only significant prognostic variable at univariate analysis was the risk group: 3-year EFS was $91.7 \%$ for low risk, $91.2 \%$ for intermediate risk, and $74.4 \%$ for high risk [18].

\section{Palliative chemotherapy}

First-line treatment

In STS, doxorubicin is still considered to be the standard first-line systemic therapy (Table 2), with response rates ranging between 16 and $27 \%$ and a 


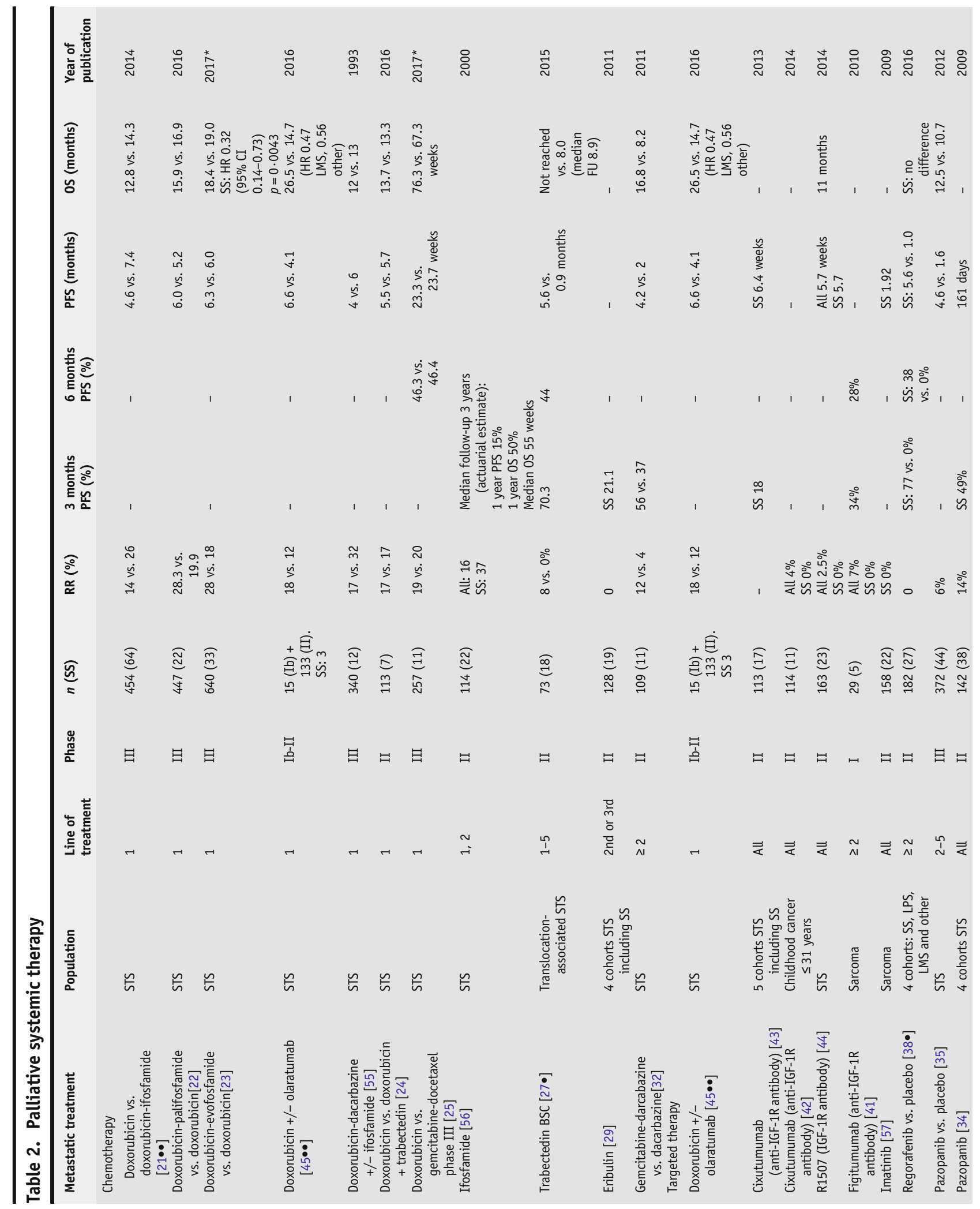




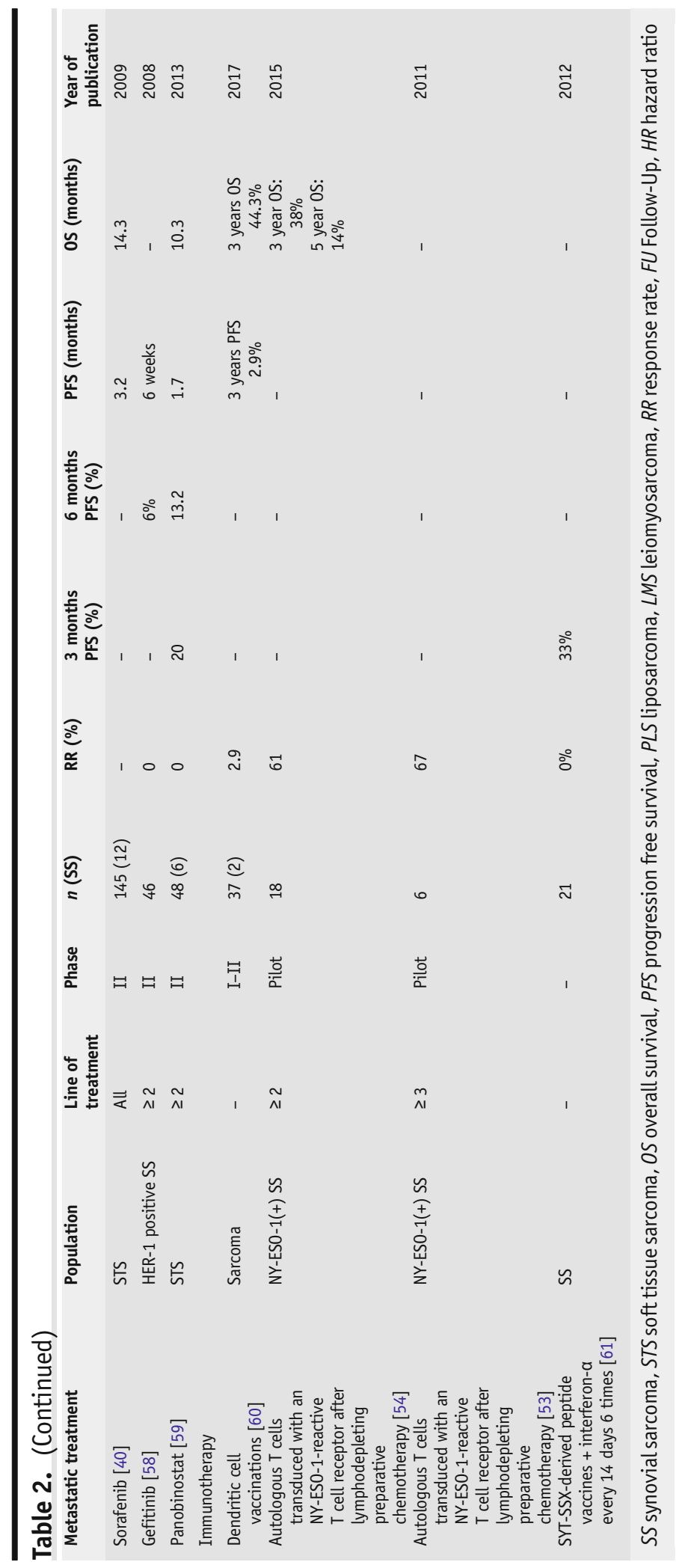


median survival in clinical studies of nowadays approximately 18 months from start of first-line systemic treatment [62]. In the METAsarc study analyzing the outcome of 2225 STS patients in the real-life setting, the OS of the 150 SS pts. who started first-line treatment was 19.7 months [63, $64 \bullet \bullet$. In 2014, the EORTC STSBG 62012 study compared single-agent doxorubicin to the combination of doxorubicin and ifosfamide in STS showing a response rate of 13.6 vs. $26.5 \%$, median progression-free survival (PFS) of 4.6 vs. 7.4 months and no significant difference in median OS (12.8 vs. 14.3 months) but significant more adverse events in the combi-

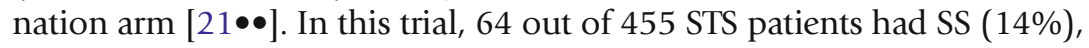
but no histotype subgroup analysis was done.

As mentioned before, SS are considered to be more chemosensitive as compared to other STS histologies [7]. Sleijfer et al. reviewed ifosfamide in different EORTC studies and found an increased response rate of ifosfamide in SS compared to other histologies [11]. A recent EORTC review of 15 clinical STS trials investigated the outcome of chemotherapy in advanced SS patients, and included 313 SS patients out of 3330 STS patients. Nine out of these 15 trials investigated anthracyclines as monotherapy arm ( $n=121$ SS), 5 a combination of doxorubicin and ifosfamide arm $(n=112$ SS), and 3 an ifosfamide monotherapy arm ( $n=42$ SS). The median PFS was significantly higher for SS patients compared to STS patients (6.3 vs. 3.7 months), as was the median OS ( 15.0 vs. 11.7 months) and the response rate (27.8 vs. 18.8\%). Comparison of the response rates (combined complete (CR) and partial response (PR)) to the different chemotherapy schedules showed a response rate of $21.5 \%$ for anthracyclines alone, 32.2\% for doxorubicin-ifosfamide, and 33\% for ifosfamide alone. It was concluded that SS patients show superior responses to chemotherapy compared to STS patients and that, compared to anthracyclines alone, ifosfamide was (although not significant) the most active drug [9•]. Recently, two large phase III trials in STS patients testing new ifosfamiderelated compounds, namely palifosfamide and evofosfamide, in combination with doxorubicin vs. doxorubicin alone, reported negative results $[22,23]$. Both trials included 5\% SS patients, but unfortunately reported no further details of this subgroup. Furthermore, a randomized phase II trial for doxorubicin plus trabectedin vs. doxorubicin alone in the first line was negative in 115 STS patients, of whom only 7 was with synovial sarcoma [24]. Very recently, a direct comparison between first-line doxorubicin and gemcitabine-docetaxel was made by Seddon et al. [25]. Five patients with synovial sarcoma out of 129 STS patients were treated with doxorubicin compared to 6 out of 128 patients treated with gemcitabine-docetaxel. After a median follow-up of 22 months, the median PFS of the total study populations was 23.3 vs. 23.7 weeks (HR 1.28, 95\% CI $0.99-1.65, p=$ 0.06 ). The proportion of patients alive and progression-free at 24 weeks did not differ between those who received doxorubicin vs. those who received gemcitabine and docetaxel (46.3 vs. $46.4 \%$ ). The number of synovial sarcoma patients in this study was too low to draw any significant conclusions.

In conclusion, in fit patients, data suggest that SS patients might benefit from first-line combination treatment of ifosfamide with doxorubicin over monotherapy doxorubicin, although-due to the rarity of the disease-this conclusion is not based on a prospective randomized study in SS. 
Several drugs are available for second-line and further treatment of advanced and metastatic STS (Table 2). The selection of a specific schedule is made on the basis of individual patient-based considerations, including age, performance score, histological subtype, tumor burden, pace of progression, main aim of treatment, toxicity experienced during first-line treatment, potential toxicity of the second-line treatment, etc. For SS, the second-line treatment of first choice for the majority of fit patients is ifosfamide, based on its known activity in SS. Alternative options are pazopanib and trabectedin in case patients are not fit enough for ifosfamide or have received the combination of doxorubicin and ifosfamide in first line. In some case, ifosfamide monotherapy can be considered after this first-line combination treatment, in particular when there has been a reasonable interval between the end of first- and start of second-line treatment. Single-center data on ifosfamide rechallenge in different STS subtypes showed the highest activity in SS [26]. The role of trabectedin in SS has been investigated in a Japanese randomized phase II study comparing trabectedin to best supportive care in translocation-related sarcomas, showed a significantly better response rate ( 8 vs. $0 \%$ ), longer median PFS (5.6 vs. 0.9 months, HR 0.07), and longer median OS (not reached vs. 8 months, HR 0.42, median follow-up 8.9 months) for the total group [27•]. Eighteen out of the total of 73 patients had SS. This subgroup had a median PFS in favor of trabectedin (HR 0.14, 95\% CI 0.03-0.68). A retrospective analysis of 61 SS patients treated with trabectedin in four European sarcoma reference centers reported a response rate of $15 \%$, a median PFS of 3 months, and a 6-month PFR of 23\% [28•]. Trabectedin in SS has never been tested in the context of a phase III trial [30]. Eribulin has also only been tested for SS in a phase II trial. This study included 128 patients in four cohorts, of which one involved the SS subtype, which included 19 patients. The primary endpoint was PFR at 12 weeks. The study was regarded positive for a cohort when the 12 -week PFR was $>40 \%$ and negative $<20 \%$. For SS, the 12 -week PFR was $21.1 \%$, for leiomyosarcoma $31.6 \%$, and for adipocyte sarcoma 46.9\% [29] The phase III trial subsequently only included leiomyosarcoma and adipocyte sarcoma [30]. Other chemotherapeutic options for STS in general include gemcitabine, dacarbazine, and cyclophosphamide-prednisone, without specific references for SS patients [31-33].

In the past years, a lot of effort has been put into the design of a more effective and less toxic treatment modalities in SS patients. At present, however, pazopanib is still the only targeted drug approved for the treatment of STS, including SS, in the advanced setting after failure of standard cytotoxic therapy. Pazopanib is an oral, multi-targeted tyrosine kinase inhibitor directed against the receptor tyrosine kinases (RTKs) vascular endothelial growth factor (VEGFR) $1 / 2 / 3$, platelet-derived growth factor (PDGFR) $\alpha / \beta$, and KIT, thereby blocking tumor growth and inhibiting angiogenesis. In the first multistrata design with pazopanib in STS, the different PFRs at 3 months were 26\% in adipocytic STS, $44 \%$, in leiomyosarcomas, $49 \%$ in SS, and 39\% in a stratum of 
other STS [34]. Based on this, the expectations for SS in the phase III study were high. The landmark PALETTE trial that led to the approval of pazopanib in STS in the advanced setting examined a total of 369 STS patients (12\% SS). Patients were randomized 2:1 to receive either pazopanib $800 \mathrm{mg}$ OD orally or placebo. Median PFS was significantly longer in the pazopanib-treated group; $4.6 \mathrm{vs.}$ 1.6 months (HR $0.31,95 \%$ CI $0.24-0.40, p<0.0001$ ), although no significant difference in median OS was detected (12.5 vs. 10.7 months, HR 0.85, 95\% CI $0.67-1.11, p=0.25)$. Partial remissions were observed in $6 \%$ of patients who received pazopanib, and another $67 \%$ had stable disease (SD) as best response [35]. For SS, the PFS and OS were promising when compared to other histologies, but due to the relatively low number of SS, no statistically significant differences were observed in this subtype. Pazopanib efficacy was also investigated in a retrospective analysis of the Named Patient Program called SPIRE study which included 24 SS patients out of 211 STS patients in total [36]. For the SS patients, the median duration on pazopanib in this study was 5.1 months and the median OS was 13.8 months. A phase I trial combining pazopanib with ifosfamide reported a PR in two patients with SS [37].

\section{Novel treatment options under development}

\section{Novel targeted therapies in the clinic}

In addition to pazopanib, various other (classes of) targeted therapy have been tested for efficacy in SS, including compounds directed against RTKs, epigenetic regulators, and immunomodulators. So far, the majority of preclinical and early promising results have failed to translate successfully to the clinic, with generally only short-lasting benefit in a small fraction of SS patients. However, some recent developments in the clinical and preclinical setting look more promising and will be further discussed.

\section{(Receptor) tyrosine kinase targeted therapies}

As the multi-RTK inhibitor pazopanib is at present, still, the only approved form of targeted therapy with evidence of clinical efficacy in SS, various studies examined the potential of targeting (other) RTKs or associated pathways in SS. To date, the highest clinical efficacy in SS has been observed for the multikinase VEGFR/PDGFR inhibitor regorafenib. The REGOSARC trial tested regorafenib $160 \mathrm{mg}$ OD for 21 of each 28 days vs. placebo in four cohorts of doxorubicin pre-treated STS patients, including 1 SS cohort [38•]. In the SS cohort, PFS was 5.6 months (95\% CI 1.4$11.6)$ with regorafenib vs. $1.0(0.8-1.4)$ with placebo (HR 0.10 (95\% CI $0.03-0.35) p<0.0001$ ) with 1 PR and 10 SDs out of 13 SS patients in the regorafenib arm. This effect was confirmed with a longer follow-up [39]. In a phase II trial with sorafenib, 12 SS out of 145 STS patients were treated with very limited efficacy [40]. Currently, a randomized EORTC phase II study ANITA comparing the oral angiogenesis inhibitor nintedanib with ifosfamide in second line is recruiting patients with advanced and metastatic STS, including SS (NCT02808247). 
A phase III trial with an SS cohort $(n=95)$ comparing in a 2:1 ratio efficacy of the multikinase VEGFR/PDGFR inhibitor anlotinib to dacarbazine is currently recruiting patients (NCT03016819). Four phase II trials with an anti-IGF1R-antibody did not show encouraging results in SS [41-44].

In the recent promising phase Ib-II study testing the combination of PDGFR antibody olaratumab plus doxorubicin vs. doxorubicin alone, only three SS patients were included [45••]. The phase III ANNOUNCE trial with similar treatment arms will hopefully have a reasonable subgroup of SS patients. Results of this study carried out in many STS subtypes, are awaited.

\section{Epigenetic modifiers}

The EZH2 inhibitor tazemetostat is currently the most studied epigenetic modulating drug in SS in the clinic. Tazemetostat activity is enhanced in integrase interactor 1 (INI1; aka SMARCB1)-negative tumors, in which INI1 loss allows EZH2 to become an oncogenic driver in tumor cells. INI1 deficiency specifically linked to the presence of the SS18-SSX1 fusion gene has been reported in SS in preclinical models [46, 47]. Accordingly, INI1 loss is a characteristic of SS tumor cell lines and tumors [48]. Preliminary data from ongoing adult phase I/II (NCT01897571) and phase II (NCT02601950) trials assessing single-agent tazemetostat activity in a variety of tumors including SS have confirmed clinical efficacy of tazemetostat in INI1-deficient tumors [49•, 50]. The three included SS patients in the phase I/II trial were, however, not INI1-negative and showed no response. As SS tumors are INI1-deficient rather than INI1-negative, cellular INI1 expression levels are reduced to varying degrees in SS, likely reflecting the variable tazemetostat treatment outcomes in SS [49•] The first results of a phase II trial with tazemetostat in SS and INI1negative tumors have been presented at the annual meeting of ASCO 2017 (NCT02601950) [50]. In 33 treated SS patients with a median of two prior systemic treatments, best response was SD in 11 patients (33\%) in which 5 patients lasted $\geq 16$ weeks. No objective responses were observed. The protocoldefined success criterion at the end of study was not met. Additional studies examining tazemetostat efficacy in SS are currently ongoing. A phase II trial with vorinostat in 40 STS patients including three SS showed no objective responses, a median PFS of 3.2 months and median OS of 12.3 months [51]. against the NY-ESO-1 cancer/testis antigen, which is expressed in $80 \%$ of SS patients, has been the most promising approach in SS in clinical testing so far [52•]. Objective responses were reported in $4 / 6$ heavily pretreated, metastatic, NY-ESO-1-expressing SS patients treated with lymphodepleting preparative chemotherapy followed by autologous $\mathrm{T}$ cells transduced with an NY-ESO-1-reactive T cell receptor, including tumor regressions. Long-term follow-up of these patients confirmed objective responses in 11/18 SS patients (61\%), including long-lasting partial and complete responses [53,54]. A subsequent multi-cohort study with an updated protocol investigated effects of genetically engineered NY-ESO-1c259T cells recognizing an NY-ESO-1 derived peptide 
complexed with HLA-A2 in advanced, NY-ESO-1-positive SS patients. So far, promising efficacy and acceptable safety data have been demonstrated, including $1 \mathrm{CR}$ and 5 PRs in the 12 treated SS patients of the first study cohort [65]. Interestingly, further data presented by D'Angelo at the annual meeting of CTOS 2017 reported responses in all four study cohorts, and the addition of fludarabine appeared to be required for optimal therapy response. Of note, in early 2016, the FDA granted breakthrough therapy designation for the affinity enhanced $\mathrm{T}$ cell therapy targeting NY-ESO for SS in the advanced setting and expressing NY-ESO-1 and HLA-A2 alleles. A variety of other clinical studies employing NY-ESO1 in SS are currently under way (e.g., NCT02457650, NCT01343043).

The results of the Alliance A091401 study with the the PD1-inhibitor nivolumab and the combination of nivolumab and the CTLA4-inhibitor ipilimumab tested in 85 STS patients, included 4 SS patients. Objective responses were reported in 5\% of patients treated with single-agent nivolumab and in $16 \%$ of the patients treated with the combination. No response was seen in SS. Median PFS and OS was 2.6 and 8.7 months for the single-agent nivolumab cohort and 4.5 and 11.2 months for the combination cohort (NCT02500797) [66••]. A second trial presented at ASCO 2017 combined low oral doses of cyclophosphamide with anti-PD1 pembrolizumab in different types of STS and GIST. In 50 evaluable patients, only three PRs were observed and very limited progression-free survival rates (NCT02406781) [67]. In the SARC028 study, pembrolizumab was administered in 85 patients with bone and soft tissue sarcoma, including 10 SS patients. For STS, median follow-up was 14.5 months. The objective response rate in the overall STS cohort was $18 \%$ and the 12 -week PFR $55 \%$. One out of 10 SS patients had a PR [68••]. An ongoing phase I-II trial combining pembrolizumab with doxorubicin in advanced or metastastic STS is currently recruiting patients (NCT02888665), as is a phase II study of Talimogene Laherparepvec (T-VEC) combined with pembrolizumab (NCT03069378). In the neoadjuvant setting, anti-PD-L1 (Durvalumab/MEDI4736) plus anti-CTLA-4 (Tremelimumab) and radiation is tested for high-risk STS (NCT03116529). The combination of RTK inhibitor axitinib and pembrolizumab is currently tested in specific types of STS including SS, after failure of anthracyclines (NCT02636725). As a first-line therapy, the combination of trabectedin, nivolumab, and anti-CTLA-4 ipilimumab is under investigation in a dose-finding phase I-II study for advanced STS (NCT03138161). A phase I trial in STS including SS with decitabine and autologous cancer testis antigen-specific dendritic cell vaccine has recently completed accrual (NCT01241162).

In conclusion, although it is too early to conclude on the efficacy of immunotherapy in SS, therapy based on engineered T cells showed exciting early

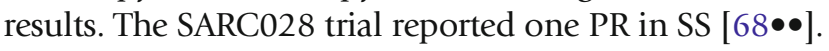

\section{Promising preclinical targeted therapies}

Although as mentioned above, various preclinical studies have failed to translate into a significant benefit for SS patients, there are some remarks to make concerning both the set-up of these studies, as well as on the types of therapies included. Most preclinical studies performed in SS to date that failed to show a benefit in the clinic, were focused on drugs 
specifically aimed at (single) RTKs. This includes research on for example the targeting of the RTK IGF-1R with monoclonal antibodies, in which preclinical promising data did not translate into encouraging results in the subsequently executed four phase II clinical trials [41-44]. On reflection, this is not necessarily an unexpected result, as it has since been shown that SS only rarely depend on a single RTK for growth and harbor little to no recurrent mutations that could be associated with a driver signature $[69 \bullet, 70 \bullet$. However, when combined with biomarkers capable of adequately identifying driver (RTK) signatures, implementation of such treatments could potentially translate into a clinical benefit in preselected subgroups of patients with a positive biomarker. The potential and proofof-concept of such an approach was published recently, in which a global phosphoproteomics approach was used to identify activated targets and pathways in sarcoma. This approach identified the RTKs ALK and MET to be the drivers of 2/4 included SS cell lines, irrespective of the SS18-SSX driver, and pharmacological inhibition of these targets with clinically relevant inhibitors-such as crizotinib-dramatically affected SS tumor growth in vitro, and even led to complete tumor regressions in vivo. As in clinical SS specimens, these key driver signatures were also detected, albeit only in a small subset of patients, these (types of) therapies are likely only beneficial to a small subset of SS patients [69•]. In this light, other lines of (preclinical) research have aimed at ways to either directly or indirectly target the known driver characterizing SS, the SS18-SSX fusions. As SS18-SSX fusion genes have been shown to affect gene expression in an epigenetic fashion, the use of epigenetic-modifying compounds have been investigated in SS [71, 72]. Preclinically, the HDAC inhibitors vorinostat and quisinostat, and the EZH2 inhibitor tazemetostat were shown to specifically target SS in an SS18-SSXdependent fashion [73-75]. Indeed, in the clinic, tazemetostat did show efficacy in SS patients as pointed out in the sections above, supporting the rationale to further investigate (combined) treatment regimens with tazemetostat, but also underlining the need to investigate the clinical potential of (other) epigenetic modifiers in SS. As mentioned before, the phase II study of vorinostat in refractory STS did not report an objective response in the three included SS patients [51], but a combination with for example the proteasome inhibitor bortezomib showed synergistic efficacy in SS models in vitro and in vivo and might be interesting [73]. Also, very recently, a synthetic lethal interaction was reported between the presence of the SS18-SSX fusion oncogene and sensitivity to inhibitors directed against the DNA damage response (DDR) kinase, ATR [76••]. Synergistic anti-tumor effects were further observed when combining the clinically relevant ATR inhibitor VX970 with PARP inhibitors or cisplatin, of which the latter combination was further examined in vivo and indeed enhanced SS tumor growth inhibition. As it was suggested that the presence of SS18-SSX fusions might introduce a vulnerability of these tumors to DDR inhibitors in general by creating a replication fork stress phenotype, and various DDR inhibitors are currently in clinical testing for other malignancies with encouraging results in certain histologies, this represents an interesting line of investigation that warrants further preclinical and clinical examination for SS [77]. In line with this hypothesis, 


\section{Conclusion}

specific sensitivity of SS cell lines to a variety of PARP inhibitors, including the clinically relevant compounds olaparib, talazoparib. and

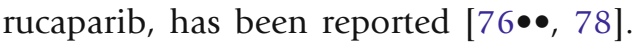

Synovial sarcoma is an intriguing disease, which is relatively chemosensitive to standard first-line treatment and pazopanib and in a subset possibly also to other RTK inhibitors. It is still early days for epigenetic modulators, and immunotherapy, but successes are observed. Based on preclinical research, DDR inhibitors should also be tested in clinical studies.

\section{Acknowledgments}

This is a summary of independent research supported by the National lnstitute for Health Research (NIHR) Biomedical Research Centre at The Royal Marsden NHS Foundation Trust and The lnstitute of Cancer Research. The views expressed are those of the author(s) and not necessarily those of the NHS, the NIHR or the Department of Health.

\section{Compliance with Ethical Standards}

\section{Conflict of interest}

Ingrid M.E. Desar has served on advisory boards for Lilly and Eisai. Emmy D.G. Fleuren declares that she has no conflict of interest. Winette T.A. van der Graaf has received research funding through a grant from Novartis.

\section{Human and animal rights and informed consent}

All reported studies/experiments with human or animal subjects performed by the authors have been previously published and complied with all applicable ethical standards (including the Helsinki declaration and its amendments, institutional/national research committee standards, and international/national/institutional guidelines).

\section{References and Recommended Reading}

Papers of particular interest, published recently, have been highlighted as:

- Of importance

$\bullet \quad$ Of major importance

1. Sultan I, Rodriguez-Galindo C, Saab R, Yasir S, Casanova $\mathrm{M}$, Ferrari A. Comparing children and adults with synovial sarcoma in the Surveillance, Epidemiology, and End Results Program, 1983 to 2005: an analysis of 1268 patients. Cancer. 2009;115(15):3537-47.
2. Corey RM, Swett K, Ward WG. Epidemiology and survivorship of soft tissue sarcomas in adults: a national cancer database report. Cancer Med. 2014;3(5):1404-15.

3.• Vlenterie M, Ho VK, Kaal SE, Vlenterie R, Haas R, van der Graaf WT. Age as an independent prognostic factor 
for survival of localized synovial sarcoma patients. Br J Cancer. 2015;113(11):1602-6.

This paper shows that for primary synovial sarcoma of comparable risk group and treated with surgery alone, the survival lowers significantly with age.

4. Wang S, Song R, Sun T, Hou B, Hong G, Mallampati S, et al. Survival changes in patients with synovial sarcoma, 1983-2012. J Cancer. 2017;8(10):1759-68.

5. Smith ME, Fisher C, Wilkinson LS, Edwards JC. Synovial sarcoma lack synovial differentiation. Histopathology. 1995;26(3):279-81.

6. Thway K, Fisher C. Synovial sarcoma: defining features and diagnostic evolution. Ann Diagn Pathol.

2014;18(6):369-80.

7. Krieg AH, Hefti F, Speth BM, Jundt G, Guillou L, Exner UG, et al. Synovial sarcomas usually metastasize after $>5$ years: a multicenter retrospective analysis with minimum follow-up of 10 years for survivors. Ann Oncol. 2011;22(2):458-67.

8. Lagarde P, Przybyl J, Brulard C, Perot G, Pierron G, Delattre $\mathrm{O}$, et al. Chromosome instability accounts for reverse metastatic outcomes of pediatric and adult synovial sarcomas. J Clin Oncol. 2013;31(5):608-15.

9.• Vlenterie M, Litiere S, Rizzo E, Marreaud S, Judson I, Gelderblom H, et al. Outcome of chemotherapy in advanced synovial sarcoma patients: review of 15 clinical trials from the European Organization for Research and Treatment of Cancer-Soft Tissue and Bone Sarcoma Group; setting a new landmark for studies in this entity. Eur J Cancer (Oxford, England). 2016;58:62-72.

This paper provide retrospective results of a large number of metastatic SS patients treated in 15 different trials vs. all other STS and shows that SS is more chemosensitive than other STS with better PFS and OS.

10. Amankwah EK, Conley AP, Reed DR. Epidemiology and therapies for metastatic sarcoma. Clin Epidemiol. 2013;5:147-62.

11. Sleijfer S, Ouali M, van Glabbeke M, Krarup-Hansen A, Rodenhuis S, Le Cesne A, et al. Prognostic and predictive factors for outcome to first-line ifosfamide-containing chemotherapy for adult patients with advanced soft tissue sarcomas: an exploratory, retrospective analysis on large series from the European Organization for Research and Treatment of Cancer-Soft Tissue and Bone Sarcoma Group (EORTC-STBSG). Eur J Cancer (Oxford, England). 2010;46(1):72-83.

12. Van Glabbeke M, Verweij J, Judson I, Nielsen OS. Progression-free rate as the principal end-point for phase II trials in soft-tissue sarcomas. Eur J Cancer (Oxford, England). 2002;38(4):543-9.

13. Saponara M, Stacchiotti S, Casali PG, Gronchi A (Neo)adjuvant treatment in localized soft tissue sarcoma: the unsolved affair. Eur J Cancer (Oxford, England). 2017;70:1-11.

14. Gronchi A, Maki RG, Jones RL. Treatment of soft tissue sarcoma: a focus on earlier stages. Future Oncol (Lond). 2017;13(1 s):13-21.
15.• Gronchi A, Ferrari S, Quagliuolo V, Broto JM, Pousa AL, Grignani G, et al. Histotype-tailored neoadjuvant chemotherapy vs. standard chemotherapy in patients with high-risk soft-tissue sarcomas (ISG-STS 1001): an international, open-label, randomized, controlled, phase 3, multicentre trial. Lancet Oncol. 2017;18(6):812-22.

This is a large study with a distinct SS stratum, reporting outcome data of neoadjuvant standard combination vs. histology-tailored chemotreatment in high risk extremity and chest wall STS.

16. Woll PI, Reichardt P, Le Cesne A, Bonvalot S, Azzarelli A, Hoekstra HJ, et al. Adjuvant chemotherapy with doxorubicin, ifosfamide, and lenograstim for resected soft-tissue sarcoma (EORTC 62931): a multicentre randomized controlled trial. Lancet Oncol. 2012;13(10):1045-54.

17. Tanaka K, Mizusawa J, Fukuda H, Araki N, Chuman H, Takahashi M, et al. Perioperative chemotherapy with ifosfamide and doxorubicin for high-grade soft tissue sarcomas in the extremities (JCOG0304). Japn J Clin Oncol. 2015;45(6):555-61.

18. Ferrari A, De Salvo GL, Brennan B, van Noesel MM, De Paoli A, Casanova $M$, et al. Synovial sarcoma in children and adolescents: the European Pediatric Soft Tissue Sarcoma Study Group prospective trial (EpSSG NRSTS 2005). Ann Oncol. 2015;26(3):567-72.

19. Vining CC, Sinnamon AJ, Ecker BL, Kelz RR, Fraker $\mathrm{DL}$, Roses RE, et al. Adjuvant chemotherapy in resectable synovial sarcoma. J Surg Oncol. 2017;116(4):550-8.

20. Le Cesne A, Ouali M, Leahy MG, Santoro A, Hoekstra HJ, Hohenberger $P$, et al. Doxorubicin-based adjuvant chemotherapy in soft tissue sarcoma: pooled analysis of two STBSG-EORTC phase III clinical trials. Ann Oncol. 2014;25(12):2425-32.

21.• Judson I, Verweij J, Gelderblom H, Hartmann JT, Schoffski P, Blay JY, et al. Doxorubicin alone vs. intensified doxorubicin plus ifosfamide for first-line treatment of advanced or metastatic soft-tissue sarcoma: a randomized controlled phase 3 trial. Lancet Oncol. 2014;15(4):415-23.

This study addressed the question of optimal first-line chemotherapy in the metastatic setting of STS, doxorubicin or the combination of doxorubicin and ifosfamide, showing a better RR and PFS, but not OS for the combination. No separate data on SS are reported.

22. Ryan CW, Merimsky O, Agulnik M, Blay JY, Schuetze SM, Van Tine BA, et al. PICASSO III: A phase III, placebo-controlled study of doxorubicin with or without palifosfamide in patients with metastatic soft tissue sarcoma. J Clin Oncol. 2016;34(32):3898-905.

23. Tap WD, Papai Z, Van Tine BA, Attia S, Ganjoo KN, Jones RL, et al. Doxorubicin plus evofosfamide versus doxorubicin alone in locally advanced, unresectable or metastatic soft-tissue sarcoma $(\mathrm{TH}$ CR-406/SARC021): an international, multicentre, open-label, randomized phase 3 trial. Lancet Oncol. 2017;18(8):1089-103. 
24. Martin-Broto J, Pousa AL, de Las Penas R, Garcia Del Muro X, Gutierrez A, Martinez-Trufero J, et al. Randomized phase II study of trabectedin and doxorubicin compared with doxorubicin alone as first-line treatment in patients with advanced soft tissue sarcomas: a Spanish group for research on sarcoma study. J Clin Oncol. 2016;34(19):2294-302.

25. Seddon B, Strauss SJ, Whelan J, Leahy M, Woll PJ, Cowie F, et al. Gemcitabine and docetaxel versus doxorubicin as first-line treatment in previously untreated advanced unresectable or metastatic soft-tissue sarcomas (GeDDiS): a randomized controlled phase 3 trial. Lancet Oncol. 2017;18(10):1397-410.

26. Noujaim J, Constantinidou A, Messiou C, Thway K, Miah A, Benson C, et al. Successful ifosfamide rechallenge in soft-tissue sarcoma. Am J Clin Oncol. 2018;41(2):147-51.

27. Kawai A, Araki N, Sugiura H, Ueda T, Yonemoto T, Takahashi $\mathrm{M}$, et al. Trabectedin monotherapy after standard chemotherapy versus best supportive care in patients with advanced, translocation-related sarcoma: a randomized, open-label, phase 2 study. Lancet Oncol. 2015;16(4):406-16.

Randomized study after first-line therapy on the effect of trabectedine in translocation positive STS, including SS.

28. Sanfilippo R, Dileo P, Blay JY, Constantinidou A, Le Cesne A, Benson C, et al. Trabectedin in advanced synovial sarcomas: a multicenter retrospective study from four European institutions and the Italian Rare Cancer Network. Anticancer Drugs. 2015;26(6):67881.

A multicenter report on the activity of trabectedin in advance SS.

29. Schoffski P, Ray-Coquard IL, Cioffi A, Bui NB, Bauer S, Hartmann JT, et al. Activity of eribulin mesylate in patients with soft-tissue sarcoma: a phase 2 study in four independent histological subtypes. Lancet Oncol. 2011;12(11):1045-52.

30. Schoffski P, Chawla S, Maki RG, Italiano A, Gelderblom H, Choy E, et al. Eribulin versus dacarbazine in previously treated patients with advanced liposarcoma or leiomyosarcoma: a randomized, open-label, multicentre, phase 3 trial. Lancet. 2016;387(10028):1629-37.

31. Ducoulombier A, Cousin S, Kotecki N, Penel N. Gemcitabine-based chemotherapy in sarcomas: a systematic review of published trials. Crit Rev Oncol Hematol. 2016;98:73-80.

32. Garcia Del Muro X, Lopez-Pousa A, Maurel J, Martin J, Martinez-Trufero J, Casado A, et al. Randomized phase II study comparing gemcitabine plus dacarbazine versus dacarbazine alone in patients with previously treated soft tissue sarcoma: a Spanish group for research on sarcomas study. J Clin Oncol. 2011;29(18):2528-33.

33. Mir O, Domont J, Cioffi A, Bonvalot $\mathrm{S}$, Boulet B, Le Pechoux C, et al. Feasibility of metronomic oral cyclophosphamide plus prednisolone in elderly patients with inoperable or metastatic soft tissue sarcoma. Eur J Cancer (Oxford, England). 2011;47(4):515-9.

34. Sleijfer S, Ray-Coquard I, Papai Z, Le Cesne A, Scurr M, Schoffski $\mathrm{P}$, et al. Pazopanib, a multikinase angiogenesis inhibitor, in patients with relapsed or refractory advanced soft tissue sarcoma: a phase II study from the European organization for research and treatment of cancer-soft tissue and bone sarcoma group (EORTC study 62,043). J Clin Oncol. 2009;27(19):3126-32.

35. van der Graaf WT, Blay JY, Chawla SP, Kim DW, BuiNguyen B, Casali PG, et al. Pazopanib for metastatic soft-tissue sarcoma (PALETTE): a randomized, doubleblind, placebo-controlled phase 3 trial. Lancet. 2012;379(9829):1879-86.

36. Gelderblom H, Judson IR, Benson C, Merimsky O, Grignani G, Katz D, et al. Treatment patterns and clinical outcomes with pazopanib in patients with advanced soft tissue sarcomas in a compassionate use setting: results of the SPIRE study. Acta Oncol (Stockholm, Sweden). 2017;1-7.

37. Hamberg P, Boers-Sonderen MJ, van der Graaf WT, de Bruijn P, Suttle AB, Eskens FA, et al. Pazopanib exposure decreases as a result of an ifosfamide-dependent drug-drug interaction: results of a phase I study. $\mathrm{Br} \mathrm{J}$ Cancer. 2014;110(4):888-93.

38. Mir O, Brodowicz T, Italiano A, Wallet J, Blay JY, Bertucci F, et al. Safety and efficacy of regorafenib in patients with advanced soft tissue sarcoma

(REGOSARC): a randomized, double-blind, placebocontrolled, phase 2 trial. Lancet Oncol.

2016;17(12):1732-42.

This randomized phase 2 study mentions results of regorafenib in STS, including SS.

39. Kotecki N, Brodowicz T, Le Cesne A, Le Deley MC, Wallet J, Italiano A, et al. Post-cross-over activity of regorafenib (RE) in soft tissue sarcoma: analysis from the REGOSARC trial. J Clin Oncol. 2017;35(Suppl):11-052.

40. Maki RG, D'Adamo DR, Keohan ML, Saulle M, Schuetze SM, Undevia SD, et al. Phase II study of sorafenib in patients with metastatic or recurrent sarcomas. J Clin Oncol. 2009;27(19):3133-40.

41. Olmos D, Postel-Vinay S, Molife LR, Okuno SH, Schuetze SM, Paccagnella ML, et al. Safety, pharmacokinetics, and preliminary activity of the anti-IGF-1R antibody figitumumab (CP-751,871) in patients with sarcoma and Ewing's sarcoma: a phase 1 expansion cohort study. Lancet Oncol. 2010;11(2):129-35. Weigel B, Malempati S, Reid JM, Voss SD, Cho SY, Chen HX, et al. Phase 2 trial of cixutumumab in children, adolescents, and young adults with refractory solid tumors: a report from the Children"s Oncology Group. Pediatr Blood Cancer. 2014;61(3):452-6.

43. Schoffski P, Adkins D, Blay JY, Gil T, Elias AD, Rutkowski P, et al. An open-label, phase 2 study evaluating the efficacy and safety of the anti-IGF-1R antibody cixutumumab in patients with previously treated advanced or metastatic soft-tissue sarcoma or Ewing 
family of tumors. Eur J Cancer (Oxford, England). 2013;49(15):3219-28.

44. Pappo AS, Vassal G, Crowley JJ, Bolejack V, Hogendoorn PC, Chugh R, et al. A phase 2 trial of R1507, a monoclonal antibody to the insulin-like growth factor- 1 receptor (IGF-1R), in patients with recurrent or refractory rhabdomyosarcoma, osteosarcoma, synovial sarcoma, and other soft tissue sarcomas: results of a sarcoma alliance for research through collaboration study. Cancer. 2014;120(16):2448-56.

45.• Tap WD, Jones RL, Van Tine BA, Chmielowski B, Elias $\mathrm{AD}$, Adkins D, et al. Olaratumab and doxorubicin versus doxorubicin alone for treatment of soft-tissue sarcoma: an open-label phase $1 \mathrm{~b}$ and randomized phase 2 trial. Lancet. 2016;388(10043):488-97.

This study shows almost a year's benefit in OS in metastatic STS patients treated with doxorubicin and olaratumab versus doxorubicin alone, which led to conditional approval by EMA and accelerated approval by FDA. There were only few SS patients on this study.

46. Wilson BG, Wang X, Shen X, McKenna ES, Lemieux $\mathrm{ME}, \mathrm{Cho} \mathrm{YJ}$, et al. Epigenetic antagonism between polycomb and SWI/SNF complexes during oncogenic transformation. Cancer Cell. 2010;18(4):316-28.

47. Kadoch C, Crabtree GR. Reversible disruption of $\mathrm{mSWI} / \mathrm{SNF}$ (BAF) complexes by the SS18-SSX oncogenic fusion in synovial sarcoma. Cell. 2013;153(1):71-85.

48. Ito J, Asano N, Kawai A, Yoshida A. The diagnostic utility of reduced immunohistochemical expression of SMARCB1 in synovial sarcomas: a validation study. Human Pathol. 2016;47(1):32-7.

49. Schoffski P, Agulnik M, Stacchiotti S, Davis LE, Villalobos VM, Italiano A. 2017. J Clin Oncol. Phase 2 multicenter study of the EZH2 inhibitor tazemetostat in adults with synovial sarcoma (NCT02601950).35(Suppl).

First results of a phase 2 study with EZH2 inhibitor tametostat in SS (abstract).

50. Gounder MM, Schöffski P, Attia S, Italiano A, Jones R, Demetri G, et al. Phase 2 multicenter study of the EZH2 inhibitor tazemetostat in adults with INI1 negative epithelioid sarcoma (NCT02601950). J Clin Oncol. 2017;35

51. Schmitt T, Mayer-Steinacker R, Mayer F, Grunwald V, Schutte J, Hartmann JT, et al. Vorinostat in refractory soft tissue sarcomas-results of a multi-centre phase II trial of the german soft tissue sarcoma and bone tumor working group (AIO). Eur J Cancer (Oxford, England). 2016;64:74-82.

52. Dallos M, Tap WD, D'Angelo SP. Current status of engineered T-cell therapy for synovial sarcoma. Immunotherapy. 2016;8(9):1073-80.

Overview of current engineered T-cell therapy in SS.

53. Robbins PF, Morgan RA, Feldman SA, Yang JC, Sherry $\mathrm{RM}$, Dudley ME, et al. Tumor regression in patients with metastatic synovial cell sarcoma and melanoma using genetically engineered lymphocytes reactive with NY-ESO-1. J Clin Oncol. 2011;29(7):917-24.
54. Robbins PF, Kassim SH, Tran TL, Crystal JS, Morgan RA, Feldman SA, et al. A pilot trial using lymphocytes genetically engineered with an NY-ESO-1-reactive Tcell receptor: long-term follow-up and correlates with response. Clin Cancer Res. 2015;21(5):1019-27.

55. Antman K, Crowley J, Balcerzak SP, Rivkin SE, Weiss GR, Elias A, et al. An intergroup phase III randomized study of doxorubicin and dacarbazine with or without ifosfamide and mesna in advanced soft tissue and bone sarcomas. J Clin Oncol. 1993;11(7):1276-85.

56. Nielsen OS, Judson I, van Hoesel Q, le Cesne A, Keizer HJ, Blay JY, et al. Effect of high-dose ifosfamide in advanced soft tissue sarcomas. A multicentre phase II study of the EORTC Soft Tissue and Bone Sarcoma Group. Eur J Cancer (Oxford, England). 2000;36(1):61-7.

57. Chugh R, Wathen JK, Maki RG, Benjamin RS, Patel SR, Meyers PA, et al. Phase II multicenter trial of imatinib in 10 histologic subtypes of sarcoma using a Bayesian hierarchical statistical model. J Clin Oncol.

2009;27(19):3148-53.

58. Ray-Coquard I, Le Cesne A, Whelan IS, Schoffski P, Bui $\mathrm{BN}$, Verweij J, et al. A phase II study of gefitinib for patients with advanced HER-1 expressing synovial sarcoma refractory to doxorubicin-containing regimens. Oncologist. 2008;13(4):467-73.

59. Cassier PA, Lefranc A, Amela EY, Chevreau C, Bui BN, Lecesne A, et al. A phase II trial of panobinostat in patients with advanced pretreated soft tissue sarcoma. A study from the French Sarcoma Group. Br J Cancer. 2013;109(4):909-14.

60. Miwa S, Nishida H, Tanzawa Y, Takeuchi A, Hayashi K, Yamamoto N, et al. Phase 1/2 study of immunotherapy with dendritic cells pulsed with autologous tumor lysate in patients with refractory bone and soft tissue sarcoma. Cancer. 2017;123(9):1576-84.

61. Kawaguchi S, Tsukahara T, Ida K, Kimura S, Murase M, Kano M, et al. SYT-SSX breakpoint peptide vaccines in patients with synovial sarcoma: a study from the Japanese Musculoskeletal Oncology Group. Cancer Sci. 2012;103(9):1625-30.

62. Constantinidou A, van der Graaf WTA. The fate of new fosfamides in phase III studies in advanced soft tissue sarcoma. Eur J Cancer (Oxford, England). 2017;84:257-61.

63. Bae S, Crowe P, Gowda R, Joubert W, Carey-Smith R, Stalley $P$, et al. Patterns of care for patients with advanced soft tissue sarcoma: experience from Australian sarcoma services. Clin Sarcoma Res. 2016;6:11.

64.• Savina M, Le Cesne A, Blay JY, Ray-Coquard I, Mir O, Toulmonde M, et al. Patterns of care and outcomes of patients with METAstatic soft tissue SARComa in a reallife setting: the METASARC observational study. BMC Med. 2017;15(1):78.

This paper reports real-life results of over 2000 metastatic STS patients treated with different lines of chemotherapy. Data on SS are reported. 
65. Mackall C, Tap WD, Glod J, Druta M, Chow WA, Aurajo DM. Open label, non-randomized, multicohort pilot study of genetically engineered NY-ESO1c259T in HLA-A2+ patients with synovial sarcoma (NCT01343043). J Clin Oncol. 2017;37(Suppl).

66.• D'Angelo SP Mahoney MR, Van Tine BA, Atkins J, Milhem MM, Jahagirdar BN, et al. Nivolumab with or without ipilimumab treatment for metastatic sarcoma (Alliance A091401): two open-label, non-comparative, randomised, phase 2 trials. Lancet Oncol.2018 Jan 19. https://doi.org/10.1016/S1470-2045(18)30006-8.

First study with combination PDL1 inhibitor and CTLA4 antibody in sarcoma.

67. Toulmonde M, Penel N, Adam J, Chevreau C, Blay JY, Le Cesne A. Combination of pembrolizumab and metronomic cyclophosphamide in patients with advanced sarcomas and GIST: A French Sarcoma Group phase II trial. J Clin Oncol. 2017;35(Suppl).

68.• Tawbi HA, Burgess M, Bolejack V, Van Tine BA, Schuetze SM, Hu J, et al. Pembrolizumab in advanced soft-tissue sarcoma and bone sarcoma (SARC028): a multicentre, two-cohort, single-arm, open-label, phase 2 trial. Lancet Oncol. 2017;18(11):1493-501.

First study published with check point inhibitor, pembrolizumab, in STS, including SS

69. Fleuren EDG, Vlenterie M, van der Graaf WTA, Hillebrandt-Roeffen MHS, Blackburn J, Ma X, et al. Phosphoproteomic profiling reveals ALK and MET as novel actionable targets across synovial sarcoma subtypes. Cancer Res. 2017;77(16):4279-92.

Based on phosphoproteomics and tissue material it was shown that in a small subset of SS ALK and MET are targets of treatment.

70. Vlenterie M, Hillebrandt-Roeffen MH, Flucke UE, Groenen PJ, Tops BB, Kamping EJ, et al. Next generation sequencing in synovial sarcoma reveals novel gene mutations. Oncotarget. 2015;6(33):34,680-90.
This paper shows the low mutational load in SS and some novel gene mutations.

71. de Bruijn DR, Allander SV, van Dijk AH, Willemse MP, Thijssen J, van Groningen JJ, et al. The synovialsarcoma-associated SS18-SSX2 fusion protein induces epigenetic gene (de)regulation. Cancer Res. 2006;66(19):9474-82.

72. Su L, Sampaio AV, Jones KB, Pacheco M, Goytain A, Lin $\mathrm{S}$, et al. Deconstruction of the SS18-SSX fusion oncoprotein complex: insights into disease etiology and therapeutics. Cancer Cell. 2012;21(3):333-47.

73. Laporte AN, Barrott JJ, Yao RJ, Poulin NM, Brodin BA, Jones $\mathrm{KB}$, et al. HDAC and Proteasome inhibitors synergize to activate pro-apoptotic factors in synovial sarcoma. PloS One. 2017;12(1):e0169407.

74. Kawano S, Grassian AR, Tsuda M, Knutson SK, Warholic NM, Kuznetsov G, et al. Preclinical evidence of anti-tumor activity induced by EZH2 inhibition in human models of synovial sarcoma. Plos One. 2016;11(7):e0158888.

75. Kawano S, Grassian AR, Tsuda M, Knutson SK, Warholic NM, Kuznetsov G, et al. Correction: preclinical evidence of anti-tumor activity induced by EZH2 inhibition in human models of synovial sarcoma. PloS One. 2017;12(1):e0170539.

$76 . \bullet \quad$ Jones SE, Fleuren EDG, Frankum J, Konde A, Williamson CT, Krastev DB, et al. ATR is a therapeutic target in synovial sarcoma. Cancer Res. 2017;77(24):7014-26.

This paper shows for the first time the role of ATR as a potential target for the treatment of SS.

77. Brandsma I, Fleuren EDG, Williamson CT, Lord CJ. Directing the use of DDR kinase inhibitors in cancer treatment. Exp Opin Invest Drugs. 2017;26(12):1341-55.

78. Yamasaki H, Miyamoto M, Yamamoto Y, Kondo T, Watanabe T, Ohta T. Synovial sarcoma cell lines showed reduced DNA repair activity and sensitivity to a PARP inhibitor. Genes Cells. 2016;21(8):852-60. 